\title{
Simultaneous occurrence of keratocystic odontogenic tumor and ameloblastoma in the mandible: A case report
}

\author{
SHOKO GAMOH $^{1}$, HIRONORI AKIYAMA ${ }^{1}$, KAZUYA TOMINAGA $^{2}$, MASAHIRO NAKAJIMA $^{3,4}$, \\ KENJI KAKUDO $^{4}$, AKIO TANAKA ${ }^{2}$ and KIMISHIGE SHIMIZUTANI ${ }^{1}$ \\ Departments of ${ }^{1}$ Oral Radiology, ${ }^{2}$ Oral Pathology, ${ }^{3}$ Dentistry for Disability and Oral Health, and \\ ${ }^{4}$ Oral and Maxillofacial Surgery, Osaka Dental University, Osaka 540-0008, Japan
}

Received September 22, 2014; Accepted May 8, 2015

DOI: $10.3892 / \mathrm{ol} .2015 .3323$

\begin{abstract}
Keratocystic odontogenic tumors (KCOTs) and ameloblastomas are benign odontogenic tumors that primarily occur in the molar region of the mandible. However, it is uncommon for these tumors to arise simultaneously in a patient's jaw. The present study reported the diagnostic process and features of a rare case of the simultaneous occurrence of KCOT and ameloblastoma in the mandible of a 45-year-old male. Image-based diagnosis was challenging due to several conditions, including the intactness of the teeth and bone cortex as well as the sizes and locations of the lesions. Based on radiographic evidence, the patient was initially misdiagnosed and underwent a biopsy for a radicular cyst and a simple bone cyst prior to the correct diagnoses of KCOT and ameloblastoma, respectively. In addition, the present study discussed the diagnostic process of the present case and reviewed previous literature regarding the simultaneous occurrence of benign tumors of the jaw.
\end{abstract}

\section{Introduction}

Keratocystic odontogenic tumors (KCOTs) are locally aggressive benign tumors which occur in the bones of both jaws with a high recurrence rate (1). They may occur at any age with a peak in the second and third decades of life (2). The most important clinical features of KCOTs are their potential for locally destructive behavior, recurrence rate, and their tendency to multiplicity, particularly when associated with naevoid basal cell carcinoma syndrome.

Ameloblastoma is a rare, benign, slow-growing but locally invasive neoplasm of the odontogenic origin involving the

Correspondence to: Dr Shoko Gamoh, Department of Oral Radiology, Osaka Dental University, 1-5-17 Otemae, Chuo-ku, Osaka 540-0008, Japan

E-mail: shoko1105@gmail.com

Key words: ameloblastoma, keratocystic odontogenic tumor, panoramic image, computed tomography image, simultaneous occurrence of odontogenic tumors mandible (80\%) and maxilla; conservative treatment results in a high recurrence rate (3). Ameloblastoma may be characterized as solid/multicystic, extraosseous/peripheral, desmoplastic or unicystic types. Unicystic type ameloblastomas account for $5-15 \%$ of all cases, as is in the present case. The mean age of presentation for cases associated with an unerupted tooth is 16 years, as opposed to 35 years in the absence of an unerupted tooth (2). It is uncommon for these tumors to simultaneously occur in a patient's jaw. To the best of our knowledge, no previous studies have reported the co-occurrence of these tumors.

Previous reports that observed the occurrence of simultaneous odontogenic lesions or simultaneous odontogenic and non-odontogenic lesions, described combined lesions, sometimes called hybrid lesions (4,5). By contrast the present study describes the case of the challenging diagnostic process of the simultaneous presentation of KCOT and ameloblastoma in the mandible of a 45-year-old male.

\section{Case report}

A 45-year-old asymptomatic male patient was referred to the outpatient clinic of the Osaka Dental University (Osaka, Japan) for further investigation of radiolucency observed on the apex of the lower-right first molar. The lesions were first detected at the local doctor whom the patient had visited for the purpose of having treated for dislocated crown in the left molar. An electric pulp test was performed and demonstrated vitality of the adjacent premolars. Clinical examination revealed no extra-oral asymmetry or swelling.

A panoramic image was obtained using conventional equipment (Super Veraview X500 AE; J Morita MFG. Corp., Kyoto, Japan) at $78 \mathrm{kV}, 9 \mathrm{~mA}$. Initial panoramic images obtained revealed the presence of a cystic lesion around the root apex of the lower right first molar whose root canal was filled. The mandibular canal was shifted downward due to the pressure from a cystic lesion. Overall, the imaging diagnosis was of a radicular cyst (Fig. 1). Radiolucency with pencil sketch-like rim was also evident in the inter-alveolar septum of the lower-right premolars; however, no resorption or adverse findings were observed and this was diagnosed as a simple bone cyst (Fig. 1). Computed tomography (CT) images were obtained using CT scanners (BrightSpeed Elite; GE Healthcare, Milwaukee, WI, 


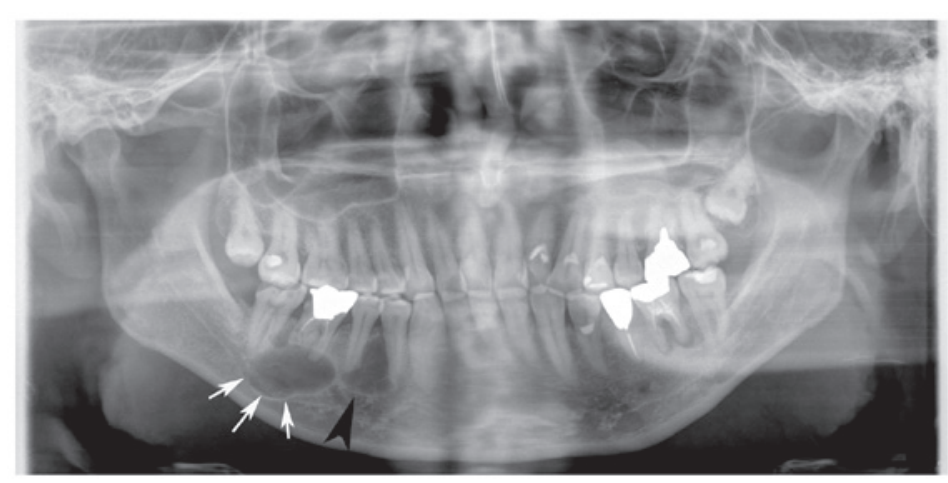

Figure 1. Initial panoramic radiograph demonstrates the presence of two round-style radiolucent lesions. One lesion was identified around the root apex of the lower-right first molar (white arrows) and another lesion was observed in the inter-alveolar septum of the lower-right premolars (black arrowhead). The imaging diagnoses of the two lesions were a radicular cyst and a simple bone cyst, respectively.

USA) at $120 \mathrm{kV}$. The electrical current was automatically optimized for the object thickness (maximum, $120 \mathrm{~mA}$ ). In addition, the $\mathrm{CT}$ was performed according to the following parameters: Slice thickness, $0.65 \mathrm{~mm}$; pitch and tube voltage, 0.625:1; and field of view, $16.8 \mathrm{~cm}^{2}$. Para-sagittal and orthogonal views (thickness, $1 \mathrm{~mm}$ ) were reformatted and reconstructed in the right molar and premolar region. These images revealed two independent abnormal lesions, comprising a 15-mm sized, round-style, well-defined cystic lesion on the root apex of the lower-right first molar and a 12-mm sized, radiolucent lesion with pencil sketch-like rim in the inter-alveolar septum of the lower-premolars (Fig. 2). These lesions induced the slight divergency of lower-right premolars. Neither of the lesions demonstrated evidence of root resorption. The wall of mandibular canal proximal to the lesion was intact and there was a slight bucco-lingual bony expansion on the lesions' medial-distal size. Therefore, the diagnoses made based on CT images were also of a radicular cyst and simple bone cyst (Fig. 2).

Following the incisional biopsy, histopathological examinations were performed, which demonstrated that each lesion had two principal features. One of the specimens revealed a thin layer of parakeratinized stratified squamous epithelium (5-8 cell layers thick) and fibrous connective tissue (Fig. 3A). The other specimen revealed an odontogenic epithelium, structured stellate reticulum and peripheral palisading within a fibrous stroma (Fig. 3B). These histopathological results indicated the diagnoses of KCOT and ameloblastoma, respectively.

Under general anesthesia, the patient underwent conservative tumor resection with extensive bone curettage associated with extractions of the lower-right molars and premolars. The diagnoses were confirmed based on excisional biopsy specimens. No recurrences or post-operative complications were observed during the 6 months follow-up period.

\section{Discussion}

The present study reported a case of simultaneous occurrence of KCOT and ameloblastoma in the mandible of a patient. To the best of our knowledge, the synchronous occurrence of KCOT and ameloblastoma as distinct lesions has not been previously reported.
In the present case, the diagnoses that were concluded from imaging and histopathological studies were entirely different. The presence of two mandibular radiolucent lesions led to the suspicion of a radicular cyst and a simple bone cyst. However, the incisional and excisional biopsies allowed for the definitive diagnosis of two pathologically distinct lesions: $\mathrm{KCOT}$ and ameloblastoma. Due to the high recurrence rate of these types of lesions $(6,7)$, close post-surgical follow-up is preferred.

In regards to the present study, it was also important to consider possible explanations for the difficult nature of the diagnosis based on radiographic images. In general, KCOT is a pathological entity with aggressive behavior and a high rate of recurrence (1). In 2005, the World Health Organization revised and updated the classification of odontogenic tumors as well as certain associated terminologies (8). Although odontogenic keratocysts were previously classified as cysts, they have been reclassified as KCOTs due to their neoplastic nature $(2,9)$. Radiologically, KCOTs appear as unilocular or multilocular radiolucencies, which commonly have a thin, reactive, sclerotic bony rim and smooth or scalloped margins; in addition, these tumors may be destructive and invade into adjacent bone (10). By contrast, ameloblastomas are slowly growing solid and cystic tumors, which are characterized as multiloculated, honeycombed, lytic lesions that are devoid of mineralization in radiographs (10).

In the panoramic images of the present case, the two lesions appeared to be unilocular radiolucent lesions without root resorption. The cystic lesion surrounding the root apex of the lower-right first molar was initially diagnosed as a radicular cyst; however, the associated lesion was ultimately revealed to be a KCOT. In addition, the radiolucency identified in the inter-alveolar septum of the lower-right premolars resembled a simple bone cyst; however, this lesion was determined to be an ameloblastoma. The CT results appeared to be concurrent with the panoramic image impression; however, this was due to a lack of reliable evidence for root resorption. As these lesions were immature, the radiographic examinations did not provide any typical characteristics that would have been suggestive of odontogenic tumors, including root resorption, divergence or multilocular radiolucency.

Retrospectively, certain imaging findings were indicative of significant pathological characteristics, including 
A

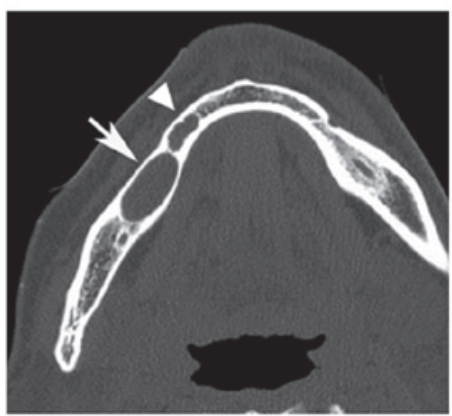

D

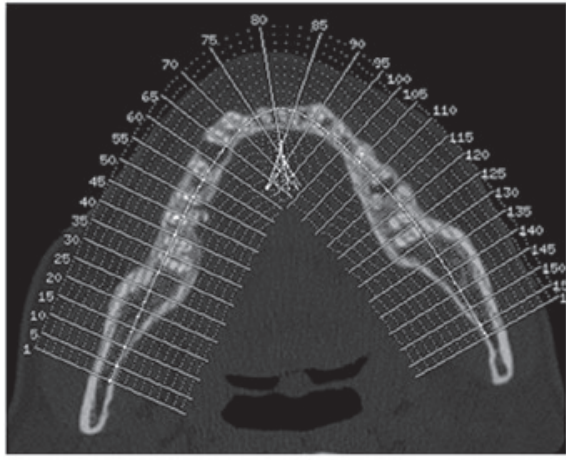

B

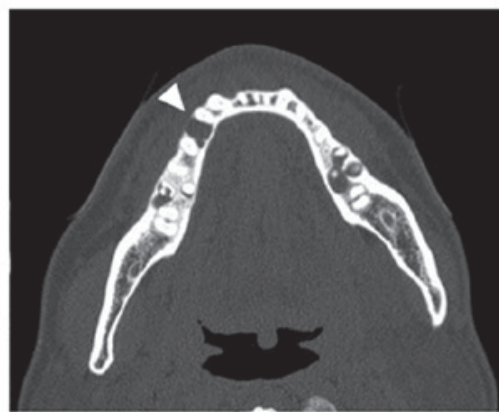

E

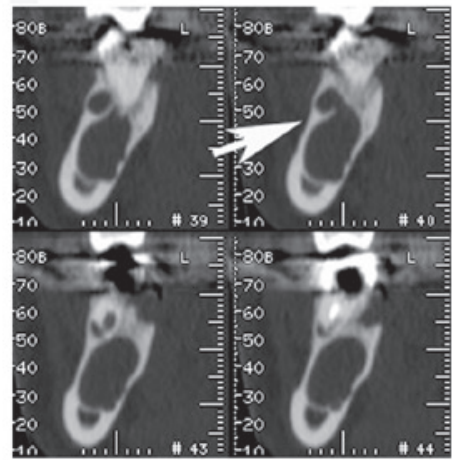

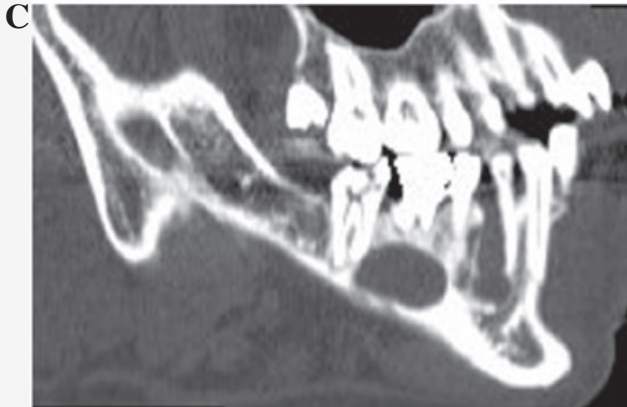

F

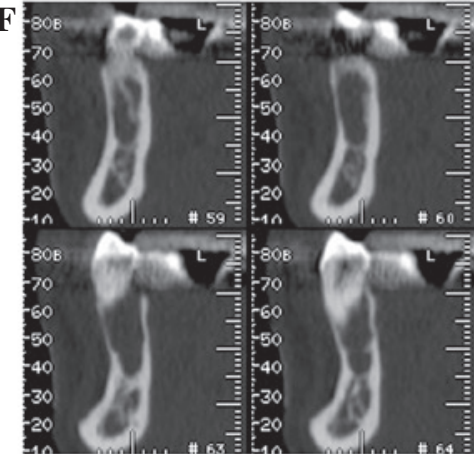

Figure 2. Computed tomography images of the patient's mandible. (A) An axial view showing the cross-section of the mandible from the patient's foot side. A 15-mm sized, round-style, well-defined cystic lesion was detected on the root apex of lower-right first molar (arrow); in addition, a 12-mm sized, radiolucent lesion was identified in the inter-alveolar septum of the lower-premolars (arrowhead). (B) An axial view demonstrated the root divergency of lower-right premolars (arrowhead). (C) A para-sagittal view revealed the septum inside the ameloblastoma as well as divergency of lower-right premolars. (D) Reference image of the orthogonal reconstruction. Numbers assigned are equivalent to the image number of each orthogonal view. (E) Orthogonal views depicting the keratocystic odontogenic tumor revealed the septum inside the KCOT (arrows). (F) Orthogonal views identifying the ameloblastoma.

A

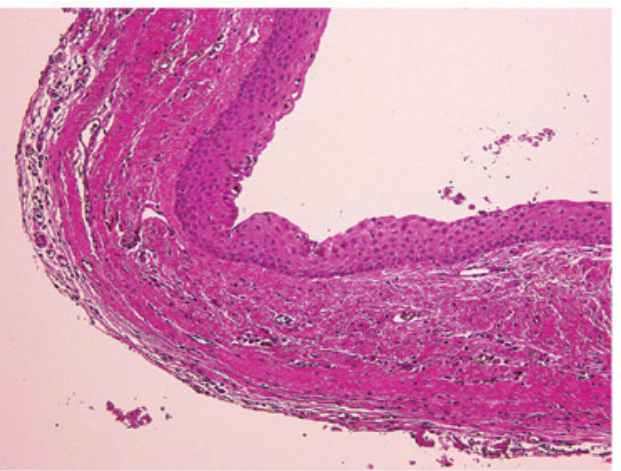

B

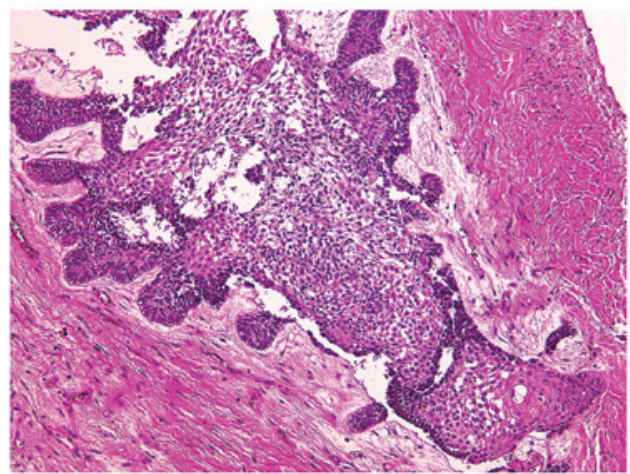

Figure 3. Histopathological analysis of the specimens obtained from the lesions following incisional biopsy. (A) Specimen from the root apex of the lower-right first molar revealed a thin layer of regularly parakeratinized stratified squamous epithelium and fibrous connective tissue. The histopathological diagnosis was of keratocystic odontogenic tumor. (B) Specimen from the inter-alveolar septum of the lower-right premolars revealed trabeculation of enamel organ-like tissue. Histopathological analysis indicated ameloblastoma. Specimens were stained with hematoxylin-eosin and images were captured at original magnification, $\mathrm{x} 20$ ).

the occurrence of a septum inside the ameloblastoma on the para-sagittal CT scan (Fig. 2C) as well as a minute septum in the KCOT on the orthogonal reconstructed CT images (Fig. 2E, arrow).

Soft X-ray is a form of X-ray with low permeability, which has a wavelength of between 10 and $102 \AA$. Soft X-ray is generally used to visualize the detailed internal structures of specimens with more precision than conventional diagnostic $\mathrm{X}$-rays. In the present study, soft X-ray images were obtained using a SOFTEX CSMW-2 system (Softex Co., Ltd, Kanagawa, Japan) in order to investigate the details of the internal structures of the lesions. As a novel trial, imaging plates were used as soft X-ray sensors, with the tube voltage set at $40 \mathrm{kV}$ and an electrical current of $2 \mathrm{~mA}$. In order to capture images of the KCOT and ameloblastoma specimens (Fig. 4A and B), the timer was set to 10 and 20 seconds, respectively. The resultant images revealed the detailed structure of the internal septum inside the lesion (Fig. 4C and D). These characteristics were concurrent with the histopathological features observed.

The occurrence of two distinct and simultaneous odontogenic lesions in one patient is extremely uncommon (6). Ameloblastomas have been previously reported to co-occur with an orthokeratinized odontogenic cyst and a glandular odontogenic cyst (10) and Fregnani et al (6) reported a case of 
Table I. Case reports of simultaneous odontogenic lesions of the jaw.

\begin{tabular}{|c|c|c|c|c|c|c|}
\hline Case (reference) & Year & Age & Gender & Histopathological diagnosis & Imaging modality & Location \\
\hline Hisatomi et al (11) & 2000 & 45 & $\mathrm{~F}$ & $\begin{array}{l}\text { Glandular odontogenic cyst and } \\
\text { ameloblastoma }\end{array}$ & $\mathrm{IO}, \mathrm{CT}, \mathrm{MR}$ & Mandible \\
\hline Fregnani et al (6) & 2006 & 21 & M & $\begin{array}{l}\text { Ameloblastoma and orthokeratinized } \\
\text { odontogenic cyst }\end{array}$ & PI, CT & Mandible \\
\hline Shimamoto et al (12) & 2011 & 48 & $\mathrm{~F}$ & $\mathrm{OF}$ and $\mathrm{KCOT}$ & PI, CT & Mandible \\
\hline Gamoh et al (13) & 2011 & 32 & $\mathrm{~F}$ & $\mathrm{OF}$ and $\mathrm{OF}$ & IO, PI, CT & Mandible \\
\hline Present case & - & 45 & M & KCOT and ameloblastoma & PI, CT, SI & Mandible \\
\hline
\end{tabular}

M, male; F, female; OF, ossifying fibroma; KCOT, keratocystic odontogenic tumor; IO, intraoral image; PI, Panoramic image; CT, computed tomography; MR, magnetic resonance imaging; SI, soft x-ray image.

A

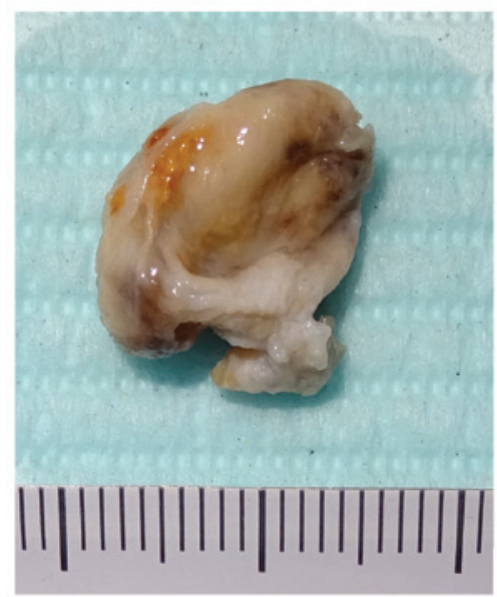

$\mathbf{C}$

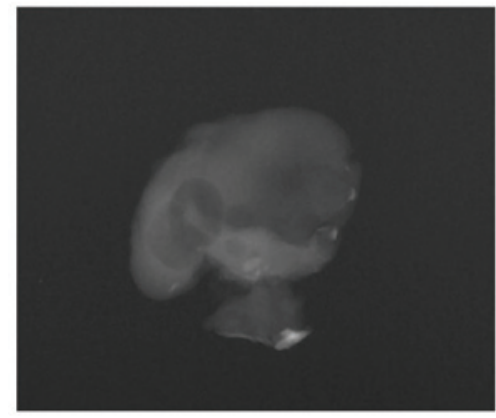

B

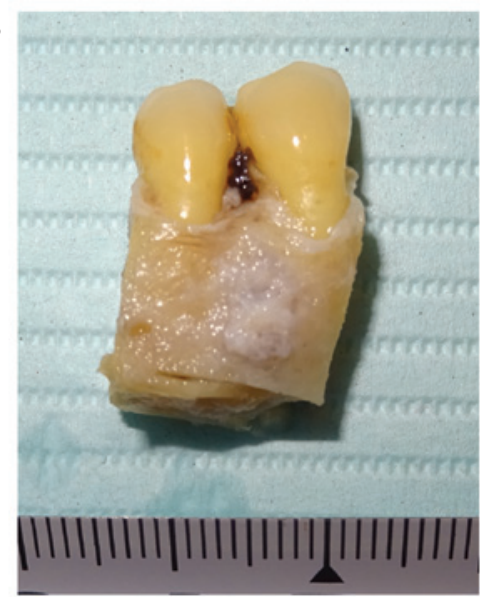

D

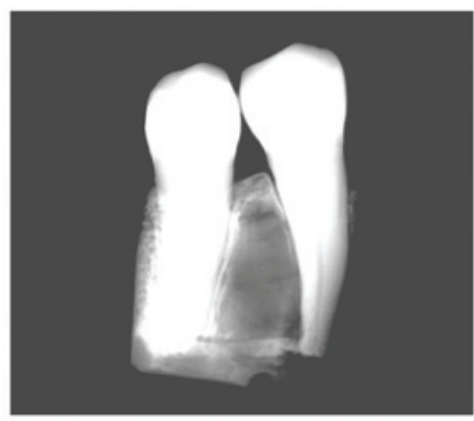

Figure 4. Soft X-ray images of the excised tumors. Images were captured of (A) the excised KCOT and (B) the ameloblastoma with adjacent teeth and alveolar bone. Soft X-ray images were then captured of (C) the KCOT specimen and (D) the ameloblastoma specimen, which demonstrated details of the internal structures of the lesions. KCOT, keratocystic odontogenic tumor.

synchronous ameloblastoma and orthokeratinized odontogenic cyst located on bilateral posterior regions of the mandible (6). In addition, Hisatomi et al (11) described a case of a glandular odontogenic cyst that was associated with an ameloblastoma; in particular, this study referred to the correlation between diagnostic imaging results and histopathological features (11). Shimamoto et al (12) presented a case of ossifying fibroma and KCOT. Another study reported a unique case of multiple ossifying fibromas in the mandible (13). Case reports of the simultaneous occurrence of odontogenic cysts and/or tumors are summarized in Table I.

KCOT is one of the consistent features of Gorlin-Goltz syndrome, which is also known as nevoid basal cell carcinoma syndrome (14). Gorlin-Goltz syndrome demonstrates an autosomal dominant inheritance pattern with variable phenotypic expression, which may result in skin, jaw and other skeletal lesions. In addition, the involvement of the central nervous system and vision in this syndrome coincide with typical facial features, including frontal bossing and hypertelorism (7). In 1965, Gorlin had already noted striking analogies between the cutaneous and jaw lesions, stating that jaw cysts correspond to the cutaneous milia and the mural proliferations observed in certain jaw cysts were analogous with adnexal skin tumors. Furthermore, the ameloblastoma may be compared with cutaneous basal-cell carcinoma (15). These previous studies therefore supported the co-occurrence of KCOT and 
ameloblastoma in the present case, although there does not appear to be any association with Gorlin-Goltz syndrome due to the lack of cutaneous milia and solitariness of the KCOT. Therefore, the lesions observed in the present study may have generated fully independently; however, the present case may offer a proof-of-principal for Gorlin's insightful theory.

The present case proved the possibility of the simultaneous occurrence of KCOT and ameloblastoma in a jaw. This knowledge should be a valuable warning as both tumors have high recurrence rate if improperly treated.

\section{Acknowledgements}

The authors would like to thank Dr Akihiro Nakajima (Second Department of Oral and Maxillofacial Surgery, Osaka Dental University) and Dr Kaname Tsuji (First Department of Oral and Maxillofacial Surgery, Osaka Dental University) for their support in the present study.

\section{References}

1. Antonoglou GN, Sándor GK, Koidou VP and Papageorgiou SN Non-syndromic and syndromic keratocystic odontogenic tumors: Systematic review and meta-analysis of recurrences. J Craniomaxillofac Surg 42: e364-e371, 2014.

2. Philipsen HP: Keratocystic odontogenic tumour. In: Pathology and Genetics of Head and Neck Tumours. Barnes L, Eveson JW, Reichart P and Sidransky D (eds). IARC Press, Lyon, pp306-307, 2005.

3. McClary AC, West RB, McClary AC, Pollack JR, Fischbein NJ, Holsinger CF, Sunwoo J, Colevas AD and Sirjani D: Ameloblastoma: A clinical review and trends in management. Eur Arch Otorhinolaryngol: Apr 30, 2015 (Epub ahead of print).

4. Siar CH and $\mathrm{Ng} \mathrm{KH}$ : 'Combined ameloblastoma and odontogenic keratocyst' or 'keratinising ameloblastoma'. Br J Oral Maxillofac Surg 31: 183-186, 1993.
5. Nishimura T, Nagakura R, Ikeda A and Kita S: Simultaneous occurrence of a squamous cell carcinoma and an ameloblastoma in the maxilla. J Oral Maxillofac Surg 58: 1297-1300, 2000.

6. Fregnani ER, Cruz Perez DE, Soares FA and Alves FA: Synchronous ameloblastoma and orthokeratinized odontogenic cyst of the mandible. J Oral Pathol Med 35: 573-575, 2006.

7. Shear M and Speight PM (eds): Odontogenic keratocyst. In: Cysts of the Oral and Maxillofacial Regions. 4th edition. Blackwell Munksgaard, Oxford, pp6-58, 2007.

8. Philipsen HP and Reichart PA: Classification of odontogenic tumours. A historical review. J Oral Pathol Med 35: 525-529, 2006.

9. Gardner DG, Heikinheimo K, Shear M, Philipsen HP and Coleman H: Ameloblastomas. In: Pathology and Genetics of Head and Neck Tumours. Barnes L, Eveson JW, Reichart P and Sidransky D (eds). International Agency for Research on Cancer (IARC) Press, Lyon, pp296-297, 2005.

10. Som PM and Curtin HD: Tumors and Tumor-like Conditions. In: Head and Neck Imaging Som PM and Brandwein MS (eds). 4th edition. Vol 1. Mosby, St. Louis, MO, pp347-352, 354-356, 2003.

11. Hisatomi M, Asaumi J, Konouchi H, Yanagi Y and Kishi K: A case of glandular odontogenic cyst associated with ameloblastoma: Correlation of diagnostic imaging with histopathological features. Dentomaxillofac Radiol 29: 249-253, 2000.

12. Shimamoto H, Kishino M, Okura M, Chindasombatjaroen J, Kakimoto N, Murakami S and Furukawa S: Radiographic features of a patient with both cemento-ossifying fibroma and keratocystic odontogenic tumor in the mandible: A case report and review of literature. Oral Surg Oral Med Oral Pathol Oral Radiol Endod 112: 798-802, 2011.

13. Gamoh S, Koseki T, Yotsui Y, Akiyama H and Shimizutani K: Mutiple ossifying fibromas of the mandible: Report of an unique case. Dental Radiology 51: 16-18, 2011.

14. Shepard M and Coleman H: Simultaneous adenomatoid odontogenic and keratocystic odontogenic tumours in a patient with Gorlin-Goltz syndrome. Aust Dent J 59: 121-124, 2014.

15. Gorlin RJ, Vickers RA, Kellen E and Williamson JJ: Multiple basal-cell nevi syndrome. An analysis of a syndrome consisting of multiple nevoid basal-cell carcinoma, jaw cysts, skeletal anomalies, medulloblastoma, and hyporesponsiveness to parathormone. Cancer 18: 89-104, 1965. 